\title{
Early Changes in the Permeability of the Blood- Brain Barrier Produced by Toxins Associated with Liver Failure
}

\author{
H. JUHLING MCCLUNG, HOWARD R. SLOAN, PRISCILLA POWERS, A. JOHN MEROLA, \\ ROBERT MURRAY, BENNY KERZNER, AND J. DENNIS POLLACK \\ Department of Pediatrics [H.J.M., H.R.S., P.P., R.M., B.K., J.D.P.], Department of Physiological Chemistry \\ [A.J.M.], and Department of Medical Microbiology and Immunology [J.D.P.], Children's Hospital of Columbus \\ and The Ohio State University College of Medicine, Columbus, Ohio 43205
}

\begin{abstract}
Our study was designed to determine whether substances that appear in the serum during the course of liver failure have a detrimental effect on the passive permeability of the blood-brain [blood-cerebrospinal fluid (CSF)] barrier. Lactic acid, octanoic acid, and ammonia were infused into rabbits for $4 \mathrm{~h}$. The permeability changes of the blood-brain barrier were quantified by infusing polyethylene glycol 400 (PEG 400) and measuring the quantity and average mol wt of the PEG 400 that entered the CSF. The lipid solubility and effective diffusional radius of the PEG molecules were also quantified to provide greater precision for measurements using this probe. None of the animals receiving toxic infusions became seriously ill during the infusions. Low dose infusions of lactic acid, octanoic acid, and ammonia increased the effective pore diameter of the blood-brain barrier from 7.3 $\AA$ to an average of $8.5 \AA$. The amount of PEG entering the CSF increased from 1.7 to $4.0(p<0.025), 4.7(p<0.025)$, and $6.7(p<0.001) \mathrm{mmol} / \mathrm{L}$, respectively. Rabbits with galactosamine-induced liver failure had $10.1 \mathrm{mmol} / \mathrm{L}$ PEG 400 in the CSF $(p>0.001)$ before any evidence of cerebral edema. These changes occur soon after these toxins accumulate in the plasma and may alone or together with other toxins account for the permeability changes that allow neurotoxic substances to enter the brain during hepatic disease and encephalopathies such as Reye's syndrome. (Pediatr Res 28: 227-231, 1990)
\end{abstract}

\section{Abbreviations}

CSF, cerebrospinal fluid

PEG, polyethylene glycol

$V_{e}$, elution volume

$\mathrm{V}_{0}$, void volume

Encephalopathy and cerebral edema are CNS manifestations of severe liver dysfunction (1). The symptoms can present acutely, as in Reye's syndrome (2) and acute liver failure (3), or they can evolve gradually during the course of chronic liver injury (4). Although many compounds such as ammonia, FFA, $\gamma$-aminobutyric acid, mercaptans, and false neurotransmitters have been proposed as the cause of the altered cerebral function in patients with severe liver dysfunction (5), few studies have

Received September 14, 1989; accepted April 26, 1990

Reprint requests: H. Juhling McClung, M.D., Columbus Children's Hospital, 700 Children's Drive, Columbus, OH 43205.

Supported by grants from the Sterling Drug Company and the Children's Hospital Research Foundation. evaluated the mechanism by which such putative toxins could cross the blood-brain and/or blood CSF barriers to gain access to vital cerebral areas (6).

We used PEG 400 as a marker of vascular pore size to quantify small changes in the passive permeability. This product is a mixture of nontoxic homologous oligomers that range in mol wt from 150 to $590 \mathrm{D}$. Because they are insoluble in lipids, they can be used to measure the intercellular channels that conduct passive water movement. After i.v. infusion of PEG, the spinal fluid can be sampled to determine the quantity and the mean mol wt, and therefore the average size, of molecules that enter the CSF through the cerebrovascular endothelium $(7,8)$. Our studies were designed to evaluate whether elevated serum levels of several toxins that appear in the serum in the course of liver failure (1) can induce deterioration in cerebrovascular-endothelial or choroid plexus functions.

\section{MATERIALS AND METHODS}

Infusion solutions. All infusion solutions were prepared in $5 \%$ dextrose with $0.45 \%$ saline. The concentration of PEG 400 (Fisher Chemical Co., Fairlawn, NJ) in the i.v. solution was 400 mM. Lactic acid, ammonium acetate, and sodium octanoate were prepared at various concentrations so that $8 \mathrm{~mL} / \mathrm{h}$ would deliver the desired number of mol/h. Sodium octanoate was dissolved in dilute $\mathrm{NaOH}$, and the solution was then back-titrated with $\mathrm{HCl}$ to a $\mathrm{pH}$ of 7.4. Each concentration of toxin was studied individually in a group of test animals. The substances infused were: lactic acid $(0.25,0.5$, and $0.75 \mathrm{mmol} / \mathrm{kg} / \mathrm{h}):$ ammonium acetate $(0.25,0.50,0.75,1.25$, and $1.50 \mathrm{mmol} / \mathrm{kg} / \mathrm{h})$, and sodium octanoate $(0.39,0.58,0.78$, and $1.14 \mathrm{mmol} / \mathrm{kg} / \mathrm{h})$. Galactosamine (Sigma Chemical Co., St. Louis, MO) was used to prepare the liver failure control group of animals. It was prepared in normal saline and administered as a $4.25 \mathrm{mmol} / \mathrm{kg}$ bolus $40 \mathrm{~h}$ before the animals were studied (9).

Animal studies. For each concentration of each toxin, we studied groups of 6-10 New Zealand White rabbits weighing approximately $2 \mathrm{~kg}$. The animals were allowed free access to water and food until the time of the study. A 22-gauge plastic needle (Angiocath, Deseret Co., Sandy, UT) was placed in a vein in each ear. Through one ear vein, PEG 400 in dextrose-saline was infused at $40 \mathrm{~mL} / \mathrm{h}$ as a $400-\mathrm{mM}$ solution. Through the opposite ear vein, one of the test toxins was infused in an identical solution at a rate of $8 \mathrm{~mL} / \mathrm{h}$. After $4 \mathrm{~h}$ of infusion, a $1-\mathrm{mL}$ blood sample was obtained via cardiac puncture and the animal was killed with a massive i.v. dose of pentobarbital. The abdomen was quickly opened, and a $30-\mathrm{mL}$ portal venous blood sample was taken for chemical determinations. The liver was promptly removed for tissue analysis and metabolic studies. Mitochondrial studies were performed immediately. The remaining portions of 
the liver were frozen in liquid nitrogen and stored at $-70^{\circ} \mathrm{C}$ for lipid analysis. A cisternal sample of CSF was obtained, and the brain was removed for evaluation of possible edema formation.

Analyses: PEG gas-liquid chromatography. Analysis for PEG oligomers was conducted as previously described (10). Briefly, $30 \mu \mathrm{L}$ of a $10-\mathrm{g} / \mathrm{L}$ solution of diethylene glycol was added to 50 $\mu \mathrm{L}$ of a CSF sample. The mixture was passed over $0.25 \mathrm{~mL}$ of a mixed-bed resin (Amberlite MB-3, Mallinckhrodt Inc., St. Louis, MO). The effluent was concentrated 10 -fold by lyophilization and injected directly onto the gas-liquid chromatograph.

Brain water content. As a measure of cerebral edema, the brain water content was determined by stripping the brain of membranes and major vessels and gently patting the surfaces dry with paper towels. One cerebral hemisphere was weighed, lyophilized, and reweighed. The loss of weight was taken as the brain water content.

Serum chemistries. Alanine aminotransferase (or serum glutamic oxaloacetic transaminase) was analyzed with commercial kits (Sigma Transaminase Kit, Sigma Chemical Co.). Analyses of blood ammonia were performed in our clinical laboratories by a colorimetric technique on the Kodak Ektochem 400 (Eastman Kodak Co., Rochester, NY). Serum osmolarities were determined by freezing-point depression (Advanced Osmometer, Advanced Instruments, Newton Highlands, MA). Blood-gas determinations were made with a blood-gas analyzer (model 175, Corning Medical, Medfield, MA). Tissue samples for light microscopy were preserved in buffered formalin. The specimens for electron microscopy were quickly diced, placed in iced glutaraldehyde, transferred to buffered osmium tetroxide, embedded, and then sliced. Lead-citrate and uranyl-acetate stains were used for examination by electron transmission microscopy.

Mitochondrial studies. To compare these data with studies of Reye's syndrome, mitochondrial respiration and the urea cycle of livers from the experimental animals were evaluated. Liver mitochondria were prepared from fresh tissue. Respiratory control and $\mathrm{P} / \mathrm{O}$ ratios of the mitochondria were assayed by polarography (11) and citrulline synthesis by an assay detailed previously (11).

Liver lipids. To determine the degree of fatty liver caused by these experimental conditions, tissue total lipids (12) and triglycerides (13) were separated and assayed (14).

Octanol:water partition coefficients. Octanol:water partition coefficients were determined to verify that PEG is primarily a water-soluble molecule. A solution of ${ }^{3} \mathrm{H}-\mathrm{PEG} 900$ (Amersham, Arlington Heights, IL) was passed over a column $(0.7 \times 240 \mathrm{~cm})$ of Biogel P-2, 200-400 mesh (Bio-Rad Laboratories, Richmond, CA) to isolate individual PEG oligomers. The purity of the individual fractions was verified by thin-layer chromatography (15). The pure tritiated oligomers, as well as samples of oligomer mixtures referred to as PEG 200, PEG 900, and PEG 4000, were added to separatory funnels that contained $5 \mathrm{~mL}$ distilled water and $5 \mathrm{~mL}$ octanol. The funnels were vigorously shaken and allowed to stand until the phases separated completely. Duplicate $1-\mathrm{mL}$ aliquots of the octanol and water layers were counted by conventional scintillation techniques with corrections for quenching by octanol. The individual oligomers tested ranged from 150 to $590 \mathrm{D}$. The partition coefficients were calculated by dividing the radioactivity in the octanol fraction by the radioactivity in the water fraction.

Effective diffusional radius of $P E G$ oligomers. To understand the membrane pore size that would be penetrated by each individual PEG oligomer, the effective diffusional radius of each individual PEG oligomer was determined. We used the technique of comparing the oligomer's elution volume on a column $(0.7 \times$ $240 \mathrm{~cm})$ of Biogel P-2 (200-400 mesh) with molecules of known size (inulin, cyanocobalamin, raffinose, sucrose, and glucose). The column was packed in $0.02 \mathrm{M}$ ammonium acetate and eluted with the same solvent. $V_{e}$ and $V_{0}$ of the column were determined with both BSA and $\mathrm{Hb}$. The $\mathrm{V}_{\mathrm{e}} / \mathrm{V}_{0}$ ratios were plotted against the logarithms of the mol wt of the test molecules (16, 17).

Data. Data were analyzed for statistical significance by the paired $t$ test. Differences were regarded as significant if $p<0.05$.

\section{RESULTS}

Blood-brain barrier permeability. For the studies evaluating PEG transfer to the CSF and changes in brain water, varying the toxin dose did not create any significant differences, so the data from all of the dosage groups for each toxin were pooled to give the results shown in Table 1. Lactic acid, octanoic acid, and ammonia increased the blood-brain barrier permeability to the PEG 400 oligomers (Table 1). In response to the administration of these toxins, there was an increase in both the mass of PEG and the mean mol wt of the FEG oligomers that entered the CSF. After the 4-h infusion of the toxic substances, the average mol wt of the PEG oligomers in the CSF increased by $13-38 \%$ (Table 1). The largest changes were observed in the animals with galactosamine-induced hepatic failure. There was a 1.2- to 2.8fold increased concentration of PEG in the CSF in each of the study groups (Table 1), whereas the galactosamine-induced liverfailure animals had an 18.5-fold increase over control values.

Clinical illnesses. Small numbers of both experimental and control group animals (three and two, respectively) died during the course of the study. Autopsies did not provide an adequate explanation for these deaths and, therefore, none of the data from these animals are reported. Only the rabbits that received the highest doses of ammonia and the most severely affected galactosamine animals became lethargic during the study period.

Cerebral edema. Cerebral edema, as manifested by an increase in brain water, was not observed in any of the experimental animals. In a separate set of experiments, for internal control purposes (data not reported), we tested the possibility that either the PEG 400 or the glucose in the infusion solutions might, by virtue of the osmoles they contribute to the infusion, partially treat the cerebral edema (18). We infused the animals with lactic acid, ammonia, or octanoic acid in the absence of PEG or in the absence of glucose. The animals in those studies were not significantly sicker (as demonstrated by clinical appearance, serum chemistry values, brain water, or PEG in the CSF) than the animals that received PEG or glucose in their infusion solutions. The serum osmolality of the animals that received no glucose infusions changed very little, suggesting that a significant portion of the serum osmolar change was due to the large glucose infusion.

Serum chemistries. There were no significant changes in serum alanine aminotransferase, $\mathrm{pH}$, bicarbonate content, or PEG concentration in response to the infusion of any of the four toxins. Moreover, the serum osmolarities were similar in all toxin groups preinfusion. The postinfusion serum osmolarities were mildly elevated in the toxin infusion groups and strikingly elevated in the galactosamine group.

Liver morphology and mitochondrial respiration. None of the experimental animals developed an abnormal liver fat content, light microscopic pattern, or mitochondrial respiration pattern. Electron microscopy revealed microvesicular fat deposition in the livers of animals that had received the high-dose (1.14 mmol/ $\mathrm{kg} / \mathrm{h}$ ) octanoate infusions.

Molecular sieve chromatography. The $\mathrm{V}_{\mathrm{e}} / \mathrm{V}_{0}$ ratios of the reference molecules, when plotted against the log of their mol wt, gave a straight line. The values for the various PEG oligomers also demonstrated a linear relationship (Fig. 1). By superimposing the two curves, as demonstrated in Figure 2, we can obtain an estimate of the effective diffusional radius for each of the individual PEG oligomer species (Table 2). This estimation allows the calculation of the increment in size between each of the PEG oligomers of $0.4 \AA$ for each additional $44 \mathrm{D}$ in mol wt. The effective diffusional pore size of the blood-brain barrier can then be estimated from the mean mol wt of the PEG oligomers 
Table 1. Mean mol wt and concentration of PEG 400 oligomers entering CSF*

\begin{tabular}{lccccc}
\hline & \multicolumn{5}{c}{ Toxin infused } \\
\cline { 2 - 6 } & Control & $\begin{array}{c}\text { Lactic } \\
\text { acid }\end{array}$ & $\begin{array}{c}\text { Octanoic } \\
\text { acid }\end{array}$ & Ammonia & Galactosamine \\
\hline Mean mol wt $( \pm$ SEM) & $257 \pm 8$ & $294 \pm 7 \ddagger$ & $291 \pm 9 \dagger$ & $306 \pm 6 \dagger$ & $355 \pm 15 \S$ \\
Effective pore size $(\AA)$ & $7.3 \pm 0.2$ & $8.4 \pm 0.2$ & $8.3 \pm 0.3$ & $8.7 \pm 0.2$ & $10.1 \pm 0.4$ \\
PEG mmol/L $( \pm$ SEM) & $1.7 \pm 0.5$ & $4.0 \pm 1.0 \dagger$ & $4.7 \pm 1.0 \dagger$ & $6.7 \pm 0.7 \S$ & $34.3 \pm 6.7 \S$ \\
Animals $(n)$ & 13 & 15 & 20 & 39 & 6 \\
\hline
\end{tabular}

* Pore sizes were calculated from the mean mol wt values of the PEG oligomers that entered the CSF.

$\dagger p<0.025$.

$\ddagger p<0.005$

$\S p<0.001$.

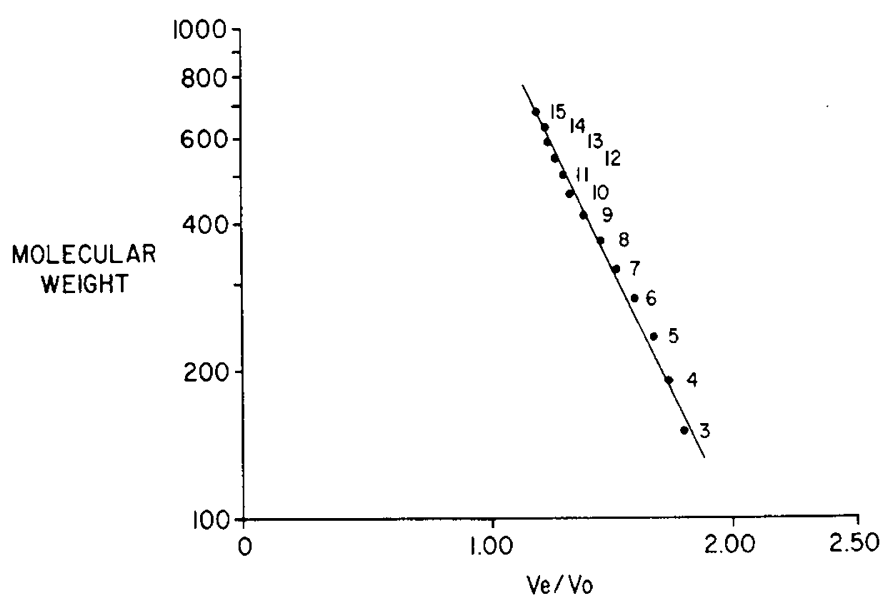

Fig. 1. Diffusion of PEG over gel columns. The diffusion rates of individual PEG oligomers demonstrate a similar rate of change (slope) when compared by mol wt.

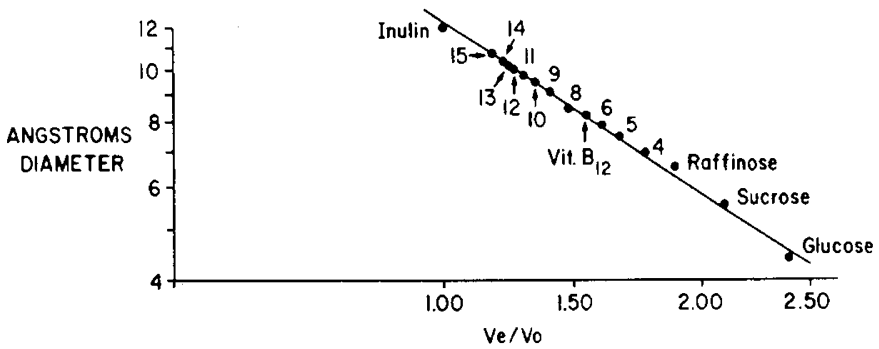

Fig. 2. Effective size (spin radius) of PEG oligomers. Diffusion data of PEG oligomers superimposed upon molecular size: diffusion rate chart of known molecules.

in a sample of CSF. For example, if the mean mol wt of the PEG oligomers in the CSF was $280 \mathrm{D}$, we would conclude that the effective pore size of the blood-brain barrier was $8 \AA$; each increase of $11 \mathrm{D}$ would suggest an increase of $0.1 \AA$ in the effective pore size.

Octanol:water ratios. If these ratios for a given set of molecules remain smaller than 0.02 , those molecules can be labeled as "water soluble" for the purpose of blood-brain barrier penetration studies. For example, drugs with values smaller than 0.02 do not effectively enter the brain. The octanol:water partition coefficient of PEG oligomer degree of polymerization 3 was less than 0.03 , and for the remainder of the oligomers (degree of polymerization 4-10), the coefficients were less than 0.02. Most values clustered between 0.015 and 0.0002 . The values for the mixtures were: 0.014 (PEG 200), 0.004 (PEG 900), and 0.00077 (PEG 4000).

\section{DISCUSSION}

The intact vascular endothelium of the CNS permits very slow movement of small polar molecules or water into the brain. As a result, the brain maintains a relatively constant chemical environment in the face of rather extreme variations that can occur in serum chemical values. Both hepatic failure and Reye's syndrome are associated with increased permeability of the blood-brain barrier to toxic substances that can enter the brain tissue. Such compounds are thought to contribute to the syndrome of hepatic encephalopathy and the associated cerebral edema (19). The purpose of our study was to determine whether these endogenous toxins contribute directly to the blood-brain barrier abnormalities observed in hepatic failure.

Zaki et al., using the Oldendorf technique, reported increases in the permeability of the blood-brain barrier in response to challenges with methyl octanoate, mercaptans, phenol, linoleic acid, and bilirubin (6). For this study, we chose as toxins those substances that commonly occur in liver failure for which there are potential therapies. We also selected a dosage that gave serum levels similar to those encountered in hepatic failure. As expected, at this low level of toxin challenges, there was no simple dose-response curve that would suggest a linear relationship between the amount of toxin in the serum and changes in the blood-brain barrier (5). Unlike many previous studies that have measured metabolic effects in animals with advanced illness (2022 ), each of these toxins caused increased permeability of the blood-brain barrier at a time when the animals did not yet appear to be severely ill.

The degree of blood-brain barrier changes seen in the animals that received lactic acid, ammonia, or octanoic acid was considerably less than that observed in the galactosamine-treated animals. These individual toxic effects may have been minimal because these challenges were very small, or because their effects were of much shorter duration than the galactosamine injury. An alternative explanation would be that, as with the coma of hepatic encephalopathy, these toxins are not individually responsible for the entire syndrome, but rather they have effects that are additive and/or synergistic (21, 23, 24).

The dramatic changes seen in the passive permeability of the blood-brain barrier in response to these toxins are in agreement

Table 2. Effective diffusional radii of $P E G$ oligomers

\begin{tabular}{lrcc}
\hline \multicolumn{1}{c}{ Name } & DP & $\begin{array}{c}\text { Mol wt } \\
\text { (D) }\end{array}$ & $\begin{array}{c}\text { Effective } \\
\text { size }(\AA)\end{array}$ \\
\hline Triethylene glycol & 3 & 150 & 6.8 \\
Tetraethylene glycol & 4 & 194 & 7.2 \\
Pentaethylene glycol & 5 & 238 & 7.6 \\
Hexaethylene glycol & 6 & 282 & 8.0 \\
Heptaethylene glycol & 7 & 326 & 8.4 \\
Octaethylene glycol & 8 & 370 & 8.8 \\
Nonaethylene glycol & 9 & 414 & 9.2 \\
Decaethylene glycol & 10 & 458 & 9.6 \\
Undecaethylene glycol & 11 & 502 & 10.0 \\
Dodecaethylene glycol & 12 & 546 & 10.4 \\
Tridecaethylene glycol & 13 & 590 & 10.8 \\
Tetradecaethylene glycol & 14 & 634 & 11.2 \\
Pentadecaethylene glycol & 15 & 698 & 11.6 \\
\hline
\end{tabular}


with the majority of the reports in the literature $(19,20,22)$. There is some controversy about the degree of blood-brain barrier effect when only short exposures to experimental probes are used (25). Our study emphasizes the need for prolonging exposure to test toxins and using a sensitive technique such as PEG 400 for evaluating the blood-brain barrier (26). An alternative explanation for the observation of quantifiable permeability changes within $4 \mathrm{~h}$ of toxin infusion would be to consider this an effect on the epithelium of the choroid plexus (the blood-cerebrospinal barrier). Although changes in the permeability of the choroid plexus are normally quantified in terms of the movement of larger molecules such as albumin or $\gamma$-globulins into the CSF, occasionally the choroid plexus has been reported to be selective for smaller molecules (27). Our study has addressed the question of the ability of toxic substances to open vascular barriers that normally protect the brain. Determining the relative penetration rates of PEG 400 through the blood-brain barrier versus the blood-CSF barrier and to what extent each of these avenues contributes to the PEG levels in the CSF will require other study techniques.

PEG 400 was selected as our probe of passive permeability of the blood-brain barrier because it is a mixture of a symmetrical series of homologous oligomers that can be accurately quantified in CSF. It has the added advantage of having very low toxicity when it is given either orally $(28,29)$ or i.v. for as long as $1 \mathrm{wk}$ (30). Moreover, PEG 400 is rapidly eliminated by the kidneys. Unfortunately, at the time our studies were conducted, the sensitivity of our assay required the administration of relatively large doses of PEG 400 to achieve quantifiable CSF levels. For comparative purposes, our dose of $8.1 \mathrm{mmol}(3.25 \mathrm{~g}) / \mathrm{kg} / \mathrm{h}$ is the equivalent on an osmolar basis of $8.1 \mathrm{mmol}(1.5 \mathrm{~g})$ of mannitol. As a result of this osmolar effect, PEG administration caused a diuresis that required the administration of large doses of i.v. fluids to keep the animals hydrated. At the conclusion of the 4$\mathrm{h}$ infusion period, both control and test animals had comparable elevations of their serum osmolalities. Such doses of PEG 400 and the resulting elevation in serum osmolalities are less than $1 / 3$ the values observed when mannitol is used to open the bloodbrain barrier artificially (31). Furthermore, the elevated serum osmolarity seen in our study had no detrimental effect on the serum or CSF values obtained in the control animals.

The octanol:water partition coefficient of PEG decreases with increasing chain length. This result indicates that long-chain PEG are more polar than their short-chain homologues. Even the shortest polymer evaluated, mol wt 150 , had a partition coefficient below 0.03 . If the lipid solubility remains below $2 \%$ of the water solubility, a molecule is a suitable probe for studies of passive permeability of membranes (32) in both adult and neonatal animals (33).

The effective molecular size of the PEG oligomers was estimated by indirect diffusion studies using molecular sieve chromatography. Because the smaller members of this polymer series are believed to be rigid, straight rods, there is no simple mathematical method to calculate their effective diffusional size. Use of the Stoke-Einstein radius equation, which uses the square root of the mol wt, requires the incorrect assumption of a globular molecular shape (34). Figure 1 demonstrates the linear relationship between the diffusion coefficient of the oligomers and the logarithm of their mol wt. It therefore appears that the PEG oligomers diffuse through the gel bed in a manner comparable to globular-shaped reference molecules that have a greater mol wt. This slower diffusion probably occurs because the linear rod shape of the PEG molecules gives them an effective spin radius comparable to that of heavier globular molecules. Our gel permeation technique allows us to estimate the relative increases in size associated with an increase in the chain length by one ethylene oxide unit of the PEG. The incremental size of $0.4 \AA$ between each oligomer is very close to the expected value for a two-carbon-oxygen unit. Using the mean mol wt of the PEG mixture to estimate the effective pore size of the membrane is a technique also validated for other chemical series (16). The pore sizes noted by this technique are quite close to the 7-9 $\AA$ estimates that Fenstermacher and Johnson (35) have made using other techniques to measure pore size in the blood-brain barrier.

Although the analytical methods for PEG 400 are difficult because they require gas-liquid chromatography, repeated lumbar spinal fluid taps do not require killing the animal for each test. This technique should allow a variety of new studies that evaluate changes in the blood-brain barrier early in the evolution of illness. Our study demonstrates that the passive permeability of the protective cerebrovascular endothelium can be significantly compromised by exposure to as little as $4 \mathrm{~h}$ of modestly elevated serum levels of lactic acid, ammonia, or FFA.

\section{REFERENCES}

1. Ede RJ, Williams R 1986 Hepatic encephalopathy and cerebral edema. Semin Liver Dis 6:107-118

2. Devivo DC, Keating JP 1976 Reye's syndrome. Adv Pediatr 22:175-229

3. Ware AI, D'Agostino A, Coombes B 1971 Cerebral edema: a major complication of massive hepatic necrosis. Gastroenterology 61:877-884

4. Gimson AES, Ede RJ, Portman B, Williams R 1986 Late onset hepatic failure. Hepatology 6:288-294

5. Zieve L 1981 The mechanism of hepatic coma. Hepatology 1:360-365

6. Zaki AEO, Warkle EN, Canalese J, Ede RJ, Williams R 1983 Potential toxins of acute liver failure and their effects on blood-brain barrier permeability. Experientia 39:988-991

7. Fenstermacher JD, Blasberg RG, Patlak CS 1981 Methods for quantifying the transport of drugs across brain barrier systems. Pharmacol Ther 14:217-248

8. Ylitalo P, Salmela P, Elo HA 1983 Correlation between brain tissue and cerebrospinal fluid concentrations of selected compounds in rats with intact and injured blood-brain barrier. Exp Neurol 81:517-527

9. Blitzer BL, Waggoner JG, Jones EA, Gralnick HR, Towne D, Butler J, Weise V, Kopin IJ, Walters I, Teychenne PF, Goodman DG, Berk PD 1978 A model of fulminant hepatic failure in the rabbit. Gastroenterology 74:664 671

10. McClung HJ, Powers PA, Sloan HR, Kerzner B 1983 A new micromethod for the quantification of low molecular weight oligomers of polyethylene glycol. Clin Chim Acta 134:245-254

11. Lutz WH, Geisbuhler TP, Pollack JD, McClung HJ, Merola AJ 1985 Inhibition of citrulline synthesis by octanoate and its modulation by adenine nucleotides. Biochem Med 34:1-10

12. Pollack JD, Cramblett HG, Flynn D, Clark D 1975 Serum and tissue lipids in Reye's syndrome. In: Pollack JD (ed) Reye's Syndrome. Grune \& Stratton, New York, pp 227-243

13. Pollack JD, Clark DS, Somerson NL 1971 Four-directional development thinlayer chromatography of lipids using trimethyl borate. J Lipid Res 12:563569

14. VanHandel E, Zilversmit DB 1957 Micromethod for the direct determination of serum triglycerides. J Lab Clin Med 50:152-157

15. Sloan HR, McClung HJ, Powers P, Kerzner B 1983 Thin-layer and gel permeation chromatographic separation of low molecular weight polyethylene glycol oligomers. Clin Chim Acta 134:255-264

16. Solomon AK 1968 Characterization of biological membranes by equivalent pores. J Gen Physiol 51:S335-S364

17. Kerzner B, Sloan HB, McClung HJ, Chidi CC, Ailabouni AH, Seckel C 1986 Absorption of glucose polymers from canine jejunum deprived of pancreatic amylase. Am J Physiol 250:G824-G829

18. Zimmerli W, Grubinger C, Tholen H, Oberholzer M, Bianchi L 1981 Mannitol treatment of cerebral edema in rats with galactosamine-induced severe hepatitis. Experientia 37:1323-1325

19. Horowitz ME, Schafer DF, Molnar P, Jones EA, Blasberg RG, Patlak CS, Waggoner J, Fenstermacher JD 1983 Increased blood-brain transfer in a rabbit model of acute liver failure. Gastroenterology 84:1003-1011

20. Livingstone AS, Potvin M, Goresky CA, Finlayson MH, Hinchey EJ 1977 Changes in the blood-brain barrier in hepatic coma after hepatectomy in the rat. Gastroenterology 73:697-704

21. Zieve L, Lyftogt C, Draves K 1983 Toxicity of a fatty acid and ammonia: interactions with hypoglycemia and Krebs cycle inhibition. J Lab Clin Med 101:930-939

22. Zaki AEO, Ede RJ, Davis M, Williams R 1984 Experimental studies of blood brain barrier permeability in acute hepatic failure. Hepatology 4:359-363

23. Zieve FJ, Zieve L, Doizaki WM, Gilsdorf RB 1974 Synergism between ammonia and fatty acids in the production of coma: implications for hepatic coma. J Pharmacol Exp Ther 191:10-16

24. Crossley IR, Wardle EN, Williams R 1983 Biochemical mechanisms of hepatic encephalopathy. Clin Sci 64:247-252

25. Lo WD, Ennis SR, Goldstein GW, McNeely DL, Betz AL 1987 The effects of galactosamine-induced hepatic failure upon blood-brain barrier permeability. Hepatology 7:452-456

26. Preston E, Haas N 1986 Defining the lower limits of blood-brain barrier permeability: factors affecting the magnitude and interpretation of permeability-area products. J Neurosci Res 16:709-716

27. Perfect JR, Savani DV, Durack DT 1986 Comparison of itraconazole and 
fluconazole in treatment of cryptococcal meningitis and candida pyelonephritis in rabbits. Antimicrob Agents Chemother 29:579-583

28. Smyth Jr HF, Carpenter CP, Weil CS 1955 The chronic oral toxicology of the polyethylene glycols. J Am Pharm Assoc 44:27-30

29. Chadwick VS, Phillips SF, Hofmann AF 1977 Measurements of intestinal permeability using low molecular weight polyethylene glycols (PEG 400). I. Chemical analysis and biological properties of PEG 400. Gastroenterology 73:241-246

30. Lockard JS, Levy RH, Congdon WC, DuCharme LL 1979 Efficacy and toxicity of the solvent polyethylene glycol 400 in monkey model. Epilepsia 20:7784

31. Mackie K, DePasquale M, Cserr HF 1986 Increased permeability of a glial blood-brain bariier during acute hyperosmotic stress. Am $\mathfrak{J}$ Physiol 251:R1186-R1192

32. Levin VA 1980 Relationship of octanol/water partition coefficient and molecular weight to rat brain capillary permeability. J Med Chem 23:682-684

33. Cornford EM, Braun LD, Oldendorf WH, Hill MA 1982 Comparison of lipidmediated blood-brain-barrier penetrability in neonates and adults. Am J Physiol 243:C161-C168

34. Sundqvist T, Magnusson KE 1985 Modelling of intestinal permeability in man to polyethylene glycols (PEG 400 and PEG 1000). Acta Physiol Scand 125:289-296

35. Fenstermacher JD, Johnson JA 1966 Filtration and reflection coefficients of the rabbit blood brain barrier. Am J Physiol 211:341-346

\section{Announcements}

\section{Annual Meeting}

The 4th annual meeting of the Sociedad Latinoamericana de Endocrinologia Pediatrica (SLEP) will be held November 4-9, 1990 in Recife, Brasil. For further information, contact: Secretario General, SLEP, Gallo 1330, 1425 Buenos Aires, Argentina, 54-1-962-4035, FAX: 54-1-962-3762.

\section{Call for Papers}

The North American Primary Care Research Group will hold its 19th annual meeting May 22-25, 1991 in Quebec, Canada. Deadline for submission of abstracts and papers is December 14, 1990. Established researchers, new investigators, practicing physicians, residents, and students are invited to submit papers. Contact NAPCRG conference secretariat at the Continuing Medical Education Office, Faculty of Medicine, Laval University, Quebec, Canada G1K 7P4, (418) 656-5958, FAX: (418) 656-3442. 